\title{
Pollution bactériologique des puits d'eau utilisés par les populations dans la Communauté Urbaine de Douala - Cameroun
}

\author{
Euphrasie DJUIKOM ${ }^{1}$, Emile TEMGOUA ${ }^{2 *}$, Louis Bertin JUGNIA ${ }^{3}$, Moïse NOLA ${ }^{4}$ et \\ Martin BAANE ${ }^{1}$ \\ ${ }^{1}$ Université de Douala, Faculté des Sciences, BP 24157 Douala, Cameroun. \\ ${ }^{2}$ Laboratoire d'Analyse des Sols et de Chimie de l'Environnement (LABASCE), Université de Dschang, BP 222 \\ Dschang, Cameroun. \\ ${ }^{3}$ Environmental Microbiology, Biotechnology Research Institute, National Research Council of Canada, 6100 \\ Royalmount Avenue, Montreal, Quebec, H4P 2R2, Canada. \\ ${ }^{4}$ Université de Yaoundé, Faculté des Sciences, BP 812 Yaoundé, Cameroun. \\ *Corresponding author, Tél: 00237770135 64, Fax: 00237334513 81, \\ E-mail:emile.temgoua@univ-dschang.com
}

\section{RESUME}

Une étude bactériologique a été menée sur 588 échantillons d'eau prélevés dans 49 puits de 7 quartiers de la ville de Douala. Les quartiers ont été choisis suivant la typologie de l'habitat. Les analyses des coliformes ont été faites suivant la technique par inclusion en une couche, et celle des streptocoques par ensemencement dans les tubes à vis avec bouillon de Rothe. Les résultats montrent que ces eaux abritent de nombreuses bactéries pathogènes, coliformes fécaux et streptocoques fécaux. Leurs densités subissent d'importantes fluctuations spatiales et temporelles, et varient entre 200 et $29 * 10^{5}$ UFC/100 ml pour les coliformes totaux et entre 30 et $44 * 10^{3} \mathrm{UFC} / 100 \mathrm{ml}$ pour les coliformes fécaux; les streptocoques fécaux sont le plus souvent présents. Les facteurs de variation sont l'environnement du puits, l'entretien, le niveau de la nappe dans le puits, la situation géographique. Les puits les plus contaminés sont ceux qui sont situés à moins de $5 \mathrm{~m}$ des sources de pollution telles que les latrines à fond perdu, les dépôts sauvages des ordures ménagères, l'absence ou le traitement régulier de l'eau, le niveau superficiel de l'eau dans le puits. Les puits aménagés, c'est-à-dire munis d'un couvercle bien utilisé et d'une margelle en béton, avec une profondeur d'au moins $4 \mathrm{~m}$, situés au moins à $15 \mathrm{~m}$ en amont d'une quelconque source de pollution et dont le traitement est régulier, présentent tous une eau de qualité beaucoup améliorée; les contaminations fécales y baissent d'au moins 1000 unités. Les points d'eau non aménagés sont très nombreux et les fortes densités de germes dans ces eaux montrent de grands risques sanitaires à court terme pour les consommateurs.

(C) 2009 International Formulae Group. All rights reserved.

Mots clés: Zone urbaine pauvre, coliformes totaux, coliformes fécaux, streptocoques fécaux, eaux de puits.

\section{INTRODUCTION}

L'approvisionnement en eau potable est un objectif qui est loin d'être atteint par toutes les populations au Cameroun. A Douala, capitale économique, la production totale d'eau du réseau public géré par la CAMWATER s'élève à peine à $80000 \mathrm{~m}^{3}$ par jour tandis que les besoins journaliers sont estimés par sa direction à environ $250000 \mathrm{~m}^{3}$. Une extension du réseau n'est pourtant pas envisagée, encore 
moins une augmentation de la capacité de production d'eau. Moins de 50\% de la population a accès au réseau conventionnel en l'an 2004 (INS, 2005). Ce faible taux de couverture oblige une grande partie de la population à recourir aux eaux souterraines (sources, puits) (Djeuda Tchapnga et al., 2001 ; Ndjama et al., 2008 ; Temgoua et al., 2009).

Les eaux souterraines représentent pour l'humanité une richesse vitale dont la vulnérabilité à la pollution microbienne est connue. La mauvaise qualité d'une eau de boisson est responsable des risques sanitaires chez les consommateurs. Les risques à long terme sont liés à la mauvaise qualité physicochimique (pollutions résultant du développement technologique et industriel) alors que ceux à court terme sont liés aux caractéristiques microbiologiques (Kreisel, 1991). L'eau en tant que réservoir de germes constitue en effet, un maillon essentiel dans la transmission des infections des voies digestives (Leclerc, 1986). Si la qualité des eaux de baignade est parfois appréciée par la recherche et l'isolement des coliphages et des bactériophages (Palmateer et al., 1991), celle des eaux de consommation humaine est régulièrement appréhendée par la recherche et l'isolement des bactéries témoins de contamination fécale (bio-indicateurs) telles que les coliformes fécaux, streptocoques fécaux, etc.

Des études sur la qualité des eaux consommées dans les ménages ont été réalisées essentiellement dans les grandes villes (Montiel, 1982 ; Niquette et al., 2001 ; Jian et al., 2005 ; Moshtaghi et Boniadian, 2007), ainsi que dans les villes sahéliennes où les quantités d'eau sont insuffisantes (Boutin, 1987 ; Ousmane et al., 2007). Peu d'études ont été menées sur la caractérisation des eaux des nappes superficielles en zone équatoriale où il y a abondance d'eau. Pourtant dans les villes de cette zone climatique, notamment dans les milieux urbaines pauvres où l'habitat est spontané, les latrines traditionnelles sont très peu profondes et côtoient les points d'eau. Il faut également noter que certaines familles vidangent leurs fosses septiques directement dans les rigoles pendant les fortes pluies. La qualité des eaux souterraines subit alors une grande variabilité (Nola et al., 1998 ; Djuikom et al., 2006 ; Temgoua et al., 2009) ; ces eaux hébergent parfois une grande communauté de bactéries coliformes, dont la concentration subit l'influence de la pluviométrie (Nola et al., 1998). Or, les ménages qui n’ont pas accès à l'eau du réseau de la CAMWATER utilisent l'eau des sources, des puits ou achètent chez les voisins ». Les municipalités voudraient s'investir dans ces méthodes alternatives d'approvisionnement en eau mais les qualités microbiologiques et physicochimiques des eaux souterraines sont encore ignorées. Cette préoccupation est d'une impérieuse nécessité, compte tenu des épidémies et même des pandémies (choléra) qui surviennent de manière récurrente dans la ville de Douala. La dernière épidémie s'est produite entre le $1^{\mathrm{er}}$ janvier et le 4 juillet 2004, au cours de laquelle 4540 cas ont été enregistrés avec plus de 25 décès (Document de travail de la Délégation de la Santé du Littoral, 2004). Ce travail préliminaire vise à déterminer la qualité bactériologique des eaux de puits de Douala, en évaluant, dans ces eaux utilisées par les populations, la distribution de quelques groupes de bactéries indicatrices de contamination fécale (coliformes fécaux et streptocoques fécaux).

\section{MATERIEL ET METHODES}

En 20 ans, la population de la ville de Douala a plus que doublé, mais la construction des infrastructures n'a pas suivi. La typologie du parcellaire de Douala établie dans les études du Schéma Directeur D'Assainissement Liquide (SDAL) de 1983 fait ressortir qu'environ 4420 ha de surface habitée sur 9800 ha de superficie totale occupée par l'habitat, sont constitués d'habitats denses ne disposant quasiment d'aucun équipement et dont l'existence est illégale. Parmi ces zones, se trouvent principalement les parties basses de la ville à proximité des drains où se concentre la majeure partie des problèmes d'insalubrité, d'accessibilité et d'insécurité. L'eau potable a souvent été citée comme le premier besoin, 
essentiellement par les femmes (Urbaplan et Sogreah, 2006). Le problème se pose parfois aussi bien en terme de quantité que de qualité. Les branchements sur le réseau de la CAMWATER ne répondent pas toujours à la demande. Les coûts de ces branchements sont d'abord considérés par certains comme chers : « Le robinet chez soi est un luxe ». De plus, la CAMWATER n'arrive pas à approvisionner tous les quartiers. Les coupures sont aussi très fréquentes.

Précisons que selon les ouvrages, les quartiers de la ville de Douala peuvent être désignés comme précaires, spontanés, non cadastrés, informels, anarchiques ou illégaux. Les quartiers retenus pour cette étude correspondent globalement à tous ces qualificatifs. Ils ont en commun d'être caractérisés par la précarité de l'habitat, par l'insécurité foncière et des conditions de vie difficiles (absence d'équipements collectifs, insalubrité, difficulté d'accès), sauf Bonamoussadi et Bonapriso où l'habitat est de type planifié.

Dans les quartiers à habitat spontané, les habitants utilisent pour la grande majorité des latrines traditionnelles de faibles profondeurs, servant en même temps de douche. Très souvent, il y a cohabitation entre les puits et les latrines en raison de l'absence d'un réseau d'assainissement collectif et du peu d'espace dont disposent les propriétaires. Le voisin creuse sa latrine sans tenir compte de la position du puits de l'autre. Ce sont ces zones de promiscuité et d'insalubrité qui constituent très souvent le point de départ des épidémies de choléra.

\section{Sites de prélèvements}

49 puits ont été décrits et leurs eaux analysées. Ces puits sont répartis dans 7 quartiers (Figure 1) de salubrité différente (Tableau 1), à raison de 7 puits par quartier. Les puits d'eau ont été choisis en fonction de leur répartition spatiale et de leur importance relative pour les populations utilisatrices; un point d'eau est d'autant plus important que le volume d'eau puisée est élevé et/ou que l'eau puisée est prioritairement destinée à l'alimentation humaine. Il faut noter que dans la majorité des cas, ces puits sont très peu profonds, et la profondeur varie entre 1,5 et $15 \mathrm{~m}$.

\section{Prélèvement et analyse des échantillons}

L'eau de chaque puits a été prélevée une fois par semaine pendant 12 semaines, de décembre 2007 à février 2008 (grande saison sèche), soit au total 588 échantillons. Les échantillons d'eau ont été prélevés dans les flacons stériles en verre, transportés en enceinte réfrigérée à $+4{ }^{\circ} \mathrm{C}$ et analysés, immédiatement dès l'arrivée au laboratoire.

Deux techniques ont été utilisées pour l'analyse de l'eau. La technique par inclusion a été choisie en utilisant la gélose Mac Conkey pour la numération des coliformes en boîtes de pétri, l'incubation se fait à $37{ }^{\circ} \mathrm{C}$ pour les coliformes totaux et à $44{ }^{\circ} \mathrm{C}$ pour les coliformes fécaux pendant $24 \mathrm{~h}$. Les résultats ont été exprimés en Unités Formatrices de Colonies (UFC) pour $100 \mathrm{ml}$ d'eau. Pour la recherche des streptocoques fécaux, la technique de présence ou absence a été utilisée en ensemençant l'échantillon d'eau dans les tubes à vis contenant le bouillon de Rothe, et en incubant à $44{ }^{\circ} \mathrm{C}$ pendant $24 \mathrm{~h}$. Une coloration de Gram est ensuite faite pour la confirmation de la présence des streptocoques fécaux à partir des tubes où le milieu est trouble (Marchal et al., 1991). Nous n'avons pas pu faire les dénombrements en raison de l'absence de matériel approprié pour cette méthode. La technique de coloration de Gram est certes présomptive mais elle permet de se prononcer sur la présence ou l'absence de streptocoques fécaux.

\section{Analyse statistique}

Les valeurs moyenne, maximale et minimale ont été ressorties de 12 analyses faites sur chaque point d'eau. Une comparaison globale de différentes moyennes a été faite grâce au test « $\mathrm{F} »$ de Fischer; la valeur limite de $\mathrm{F}$ pour laquelle les moyennes comparées diffèrent entre elles est donnée par la formule $\mathrm{F}^{\mathrm{C}-1}{ }_{\mathrm{N}-\mathrm{C}}$, où $\mathrm{C}$ représente le nombre de points d'eau et $\mathrm{N}$, le nombre total de prélèvements réalisés (Schwartz, 1984). 


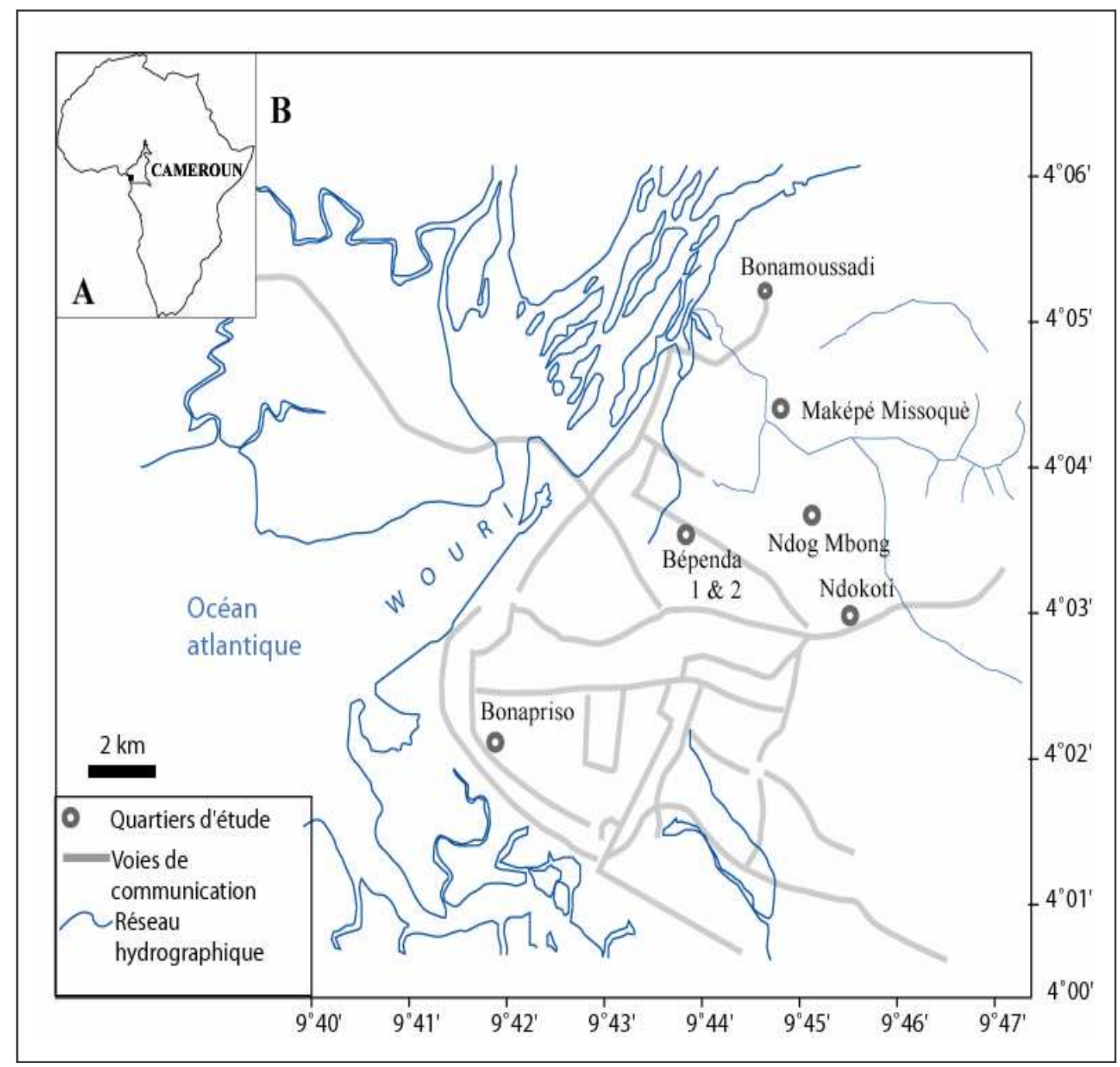

Figure 1: Carte de localisation de Douala au Cameroun et en Afrique (A) et des quartiers prospectés dans la ville de Douala (B).

\section{RESULTATS}

\section{Environnement physique des points d'eau} étudiés

La localisation géographique des différents points d'eau est donnée dans le tableau 2. Les points d'eau ont été classés en quatre groupes (A, B, C et D) en fonction de l'état de leur environnement (Tableau 3). Les distances entre les points d'eau et les habitations les plus proches varient de 1 à 10 $\mathrm{m}$. Tous les puits ne sont pas surmontés d'une margelle convenable, celle-ci peut être soit en tôles, soit en béton, d'une hauteur de 0,5 à $1 \mathrm{~m}$, ou tout simplement 2 ou 3 pneus de voiture superposés. Il existe également des couvercles pour certains puits, l'usage de ces couvercles se fait de manière négligée dans certaines concessions; le puits peut ne pas être fermé pendant des jours malgré l'existence du couvercle. Le seau de puisage est également entretenu de manière différente (négligé et déposé parfois au sol, la corde à même le sol chez certains ; ailleurs le seau de puisage n'est pas unique, chaque personne vient avec le sien. Dans les meilleurs cas, non seulement le seau est unique mais aussi il existe une poulie de 
Tableau 1: Quartiers d'échantillonnage et leur typologie.

\begin{tabular}{|c|c|}
\hline Quartiers & Typologie du quartier \\
\hline $\begin{array}{l}\text { Maképè Missoquè, } \\
\text { Bépanda 1, Bépanda } \\
\text { 2, }\end{array}$ & $\begin{array}{l}\text { quartiers très peuplés à habitat spontané, sans aucun schéma directeur de } \\
\text { lotissement et où l'accès difficile compromet la construction du réseau de } \\
\text { distribution d'eau potable. }\end{array}$ \\
\hline $\begin{array}{l}\text { Ndog-Bong, } \\
\text { Ndokoti, }\end{array}$ & $\begin{array}{l}\text { quartiers moins peuplés et organisés suivant le plan d'urbanisation de la } \\
\text { municipalité. A Ndog-Bong, presque chaque maison est dans une barrière ; } \\
\text { cependant à Ndokoti, il en est autrement et il existe des usines (fabrication de la } \\
\text { bière (GUINESS), de lait (CAMLAT), de verres (SOCAVER)) qui ne } \\
\text { disposent d'aucun système de canalisation et de traitement des eaux usées qui } \\
\text { y sont issues, ce qui signifie que ces eaux sont directement orientées dans la } \\
\text { nature. }\end{array}$ \\
\hline $\begin{array}{l}\text { Bonamoussadi, } \\
\text { Bonapriso }\end{array}$ & $\begin{array}{l}\text { quartiers de " Haut-Standing », où la propreté est l'une des priorités, et dont les } \\
\text { puits quand ils existent sont bien entretenus, mais ces quartiers subissent des } \\
\text { inondations en saison de pluies. }\end{array}$ \\
\hline
\end{tabular}

rotation à tel point qu'il n'y ait aucun contact du seau ni de la corde avec l'environnement). Les eaux de ces puits subissent des traitements périodiques selon les familles, parfois une fois par mois, ou tous les 3 mois, une fois par an, dans tous les cas à l'eau de javel, les quantités utilisées étant également variables et incontrôlées.

\section{Coliformes et streptocoques dans les eaux des puits \\ Les concentrations maximales de} coliformes totaux varient de 500 à $29 * 10^{5}$ $\mathrm{UFC} / 100 \mathrm{ml}$ (soit entre 2,70 et $6,42 \mathrm{UFC} / 100$ $\mathrm{ml}$ en valeur logarithmique, Figure 2). Les concentrations minimales vont de 100 à $96,8 * 10^{3}$ UFC/100 $\mathrm{ml}$ pour les coliformes totaux. Les plus grandes valeurs moyennes sont obtenues à Ndokoti, Maképè Missoquè et Bépanda 1 (Tableau 4). A Ndokoti, les teneurs sont très variables (Figure 2), les sources de pollution y étant diverses, liées à la présence des industries comme CAMLAIT, GUINESS, SOCAVER. Il faut noter que certaines hausses de densités bactériennes doivent être ponctuelles, cela dépendant de la nature des substances infiltrées jusqu'à la nappe phréatique, sachant que ces substances peuvent inhiber ou activer la croissance microbienne. Par ailleurs, les paramètres tels que la profondeur du puits, la fréquence de traitement, la distance par rapport à une quelconque source de pollution, peuvent induire des variations difficiles à contrôler.

Les concentrations maximales de coliformes fécaux s'échelonnent entre 30 et $44,8 * 10^{3}$ UFC/100 ml (soit entre 1,47 et 4,65 en valeur logarithmique, Figure 3). Les puits des quartiers Bépanda 1 (puits $\mathrm{B}_{3}, \mathrm{~B}_{4}, \mathrm{~B}_{6}$ et $\mathrm{B}_{7}$ ) et Maképè Missoquè $\left(M_{2}\right.$ et $\left.M_{3}\right)$ sont les plus affectés (Figure 3), avec plus de 2000 UFC/100 ml de coliformes fécaux. Aussi, ces puits sont localisés à proximité des latrines ; l'occupation anarchique des parcelles, le ruissellement des eaux d'égouts, le mauvais entretien des puits par les utilisateurs euxmêmes, la puisette qui traîne à même le sol, l'inexistence du couvercle, sont autant de facteurs liés à la hausse de densité des bactéries dans ces puits. De plus, le quartier Maképè Missoquè est une vaste zone marécageuse. Dans ces mêmes quartiers, les puits $\mathrm{B}_{1}, \mathrm{~B}_{2}, \mathrm{M}_{4}$ à $\mathrm{M}_{7}$ montrent de faible quantité de coliformes fécaux. Cela serait lié à leur situation en amont des latrines et à leur statut aménagé (Tableau 3).

Par ailleurs, la moyenne de chaque groupe de bactéries dans chaque point d'eau a été calculée. La comparaison au moyen du test « $\mathrm{F} »$ des valeurs obtenues montre que le peuplement de chaque groupe de bactéries varie significativement $(\mathrm{p}<0,01)$ d'un site à l'autre. Il en ressort, en considérant les 
E. DJUIKOM et al. / Int. J. Biol. Chem. Sci. 3(5): 967-978, 2009

Tableau 2 : Coordonnées géographiques des puits d'eau analysés ( $\mathrm{X}=$ latitude nord ; Y = longitude est).

\begin{tabular}{|c|c|c|c|c|c|c|c|c|}
\hline \multirow[t]{3}{*}{ Bépanda 1} & & B1 & B2 & B3 & B4 & B5 & B6 & B7 \\
\hline & $\mathrm{X}$ & $4^{\circ} 04^{\prime} 02,6^{\prime \prime}$ & $4^{\circ} 04^{\prime} 4,4^{\prime \prime}$ & $4^{\circ} 04^{\prime} 4,3^{\prime \prime}$ & $4^{\circ} 04^{\prime} 3,4^{\prime \prime}$ & $4^{\circ} 04^{\prime} 2,6^{\prime \prime}$ & $4^{\circ} 04^{\prime} 2,9^{\prime \prime}$ & $4^{\circ} 04^{\prime} 3,1^{\prime \prime}$ \\
\hline & $\mathrm{Y}$ & $9^{\circ} 43^{\prime} 17,5^{\prime \prime}$ & $9^{\circ} 43^{\prime} 16,5^{\prime \prime}$ & $9^{\circ} 43^{\prime} 17,1^{\prime \prime}$ & $9^{\circ} 43^{\prime} 26,5^{\prime \prime}$ & $9^{\circ} 43^{\prime} 21,2^{\prime \prime}$ & $9^{\circ} 43^{\prime} 15,8^{\prime \prime}$ & $9^{\circ} 43^{\prime} 21,2^{\prime \prime}$ \\
\hline \multirow[t]{3}{*}{ Bépanda 2} & & $\mathrm{P} 1$ & $\mathrm{P} 2$ & $\mathrm{P} 3$ & $\mathrm{P} 4$ & P5 & P6 & P7 \\
\hline & $\mathrm{X}$ & $4^{\circ} 04^{\prime} 13^{\prime \prime}$ & $4^{\circ} 04^{\prime} 22,6^{\prime \prime}$ & $4^{\circ} 04^{\prime} 14,3^{\prime \prime}$ & $4^{\circ} 04^{\prime} 15,9^{\prime \prime}$ & $4^{\circ} 04^{\prime} 32,6^{\prime \prime}$ & $4^{\circ} 04^{\prime} 25,9^{\prime \prime}$ & $4^{\circ} 04^{\prime} 33,1^{\prime \prime}$ \\
\hline & $\mathrm{Y}$ & $9^{\circ} 43^{\prime} 29,6^{\prime \prime}$ & $9^{\circ} 43^{\prime} 37,9^{\prime \prime}$ & $9^{\circ} 43^{\prime 2} 27,1^{\prime \prime}$ & $9^{\circ} 43^{\prime} 36,5^{\prime \prime}$ & $9^{\circ} 43^{\prime} 31,2^{\prime \prime}$ & $9^{\circ} 43^{\prime} 35,8^{\prime \prime}$ & $9^{\circ} 43^{\prime} 29,2^{\prime \prime}$ \\
\hline \multirow[t]{3}{*}{ Ndog-Bong } & & N1 & N2 & N3 & N4 & N5 & N6 & N7 \\
\hline & $\mathrm{X}$ & $4^{\circ} 03^{\prime} 8,8^{\prime \prime}$ & $4^{\circ} 03^{\prime} 12,1^{\prime \prime}$ & $4^{\circ} 03^{\prime} 11,7^{\prime \prime}$ & $4^{\circ} 03^{\prime} 15,1^{\prime \prime}$ & $4^{\circ} 03^{\prime} 16,7^{\prime \prime}$ & $4^{\circ} 03^{\prime} 18,6^{\prime \prime}$ & $4^{\circ} 03^{\prime} 19,3^{\prime \prime}$ \\
\hline & $\mathrm{Y}$ & $9^{\circ} 44^{\prime} 40,3^{\prime \prime}$ & $9^{\circ} 44^{\prime} 35,8^{\prime \prime}$ & $9^{\circ} 44^{\prime} 36,8^{\prime \prime}$ & $9^{\circ} 44^{\prime} 43,1^{\prime \prime}$ & $9^{\circ} 44^{\prime} 38,5^{\prime \prime}$ & $9^{\circ} 44^{\prime} 40,1^{\prime \prime}$ & $9^{\circ} 44^{\prime} 39,2^{\prime \prime}$ \\
\hline \multirow{3}{*}{$\begin{array}{l}\text { Maképé } \\
\text { Missoquè }\end{array}$} & & M1 & M2 & M3 & M4 & M5 & M6 & M7 \\
\hline & $\mathrm{X}$ & $4^{\circ} 03^{\prime} 32,2^{\prime \prime}$ & $4^{\circ} 03^{\prime} 36,2^{\prime \prime}$ & $4^{\circ} 03^{\prime} 35,8^{\prime \prime}$ & $4^{\circ} 03^{\prime} 33,8^{\prime \prime}$ & $4^{\circ} 03^{\prime} 38,6^{\prime \prime}$ & $4^{\circ} 03^{\prime} 28,9^{\prime \prime}$ & $4^{\circ} 03^{\prime} 33,1^{\prime \prime}$ \\
\hline & $\mathrm{Y}$ & $9^{\circ} 44^{\prime} 26,8^{\prime \prime}$ & $9^{\circ} 44^{\prime} 27,2^{\prime \prime}$ & $9^{\circ} 44^{\prime} 26,5^{\prime \prime}$ & $9^{\circ} 44^{\prime} 27,1^{\prime \prime}$ & $9^{\circ} 44^{\prime} 25,2^{\prime \prime}$ & $9^{\circ} 44^{\prime} 35,8^{\prime \prime}$ & $9^{\circ} 44^{\prime} 30,2^{\prime \prime}$ \\
\hline \multirow[t]{3}{*}{ Ndokoti } & & D1 & D2 & D3 & D4 & D5 & D6 & D7 \\
\hline & $\mathrm{X}$ & $4^{\circ} 02^{\prime} 21,2^{\prime \prime}$ & $4^{\circ} 02^{\prime} 19^{\prime \prime}$ & $4^{\circ} 02^{\prime} 16,16^{\prime \prime}$ & $4^{\circ} 02^{\prime} 14,9^{\prime \prime}$ & $4^{\circ} 02^{\prime} 22,6^{\prime \prime}$ & $4^{\circ} 02^{\prime} 23,9^{\prime \prime}$ & $4^{\circ} 02^{\prime} 19,6^{\prime \prime}$ \\
\hline & $\mathrm{Y}$ & $9^{\circ} 45^{\prime} 09,2^{\prime \prime}$ & $9^{\circ} 45^{\prime} 7,7^{\prime \prime}$ & $9^{\circ} 45^{\prime} 06,3^{\prime \prime}$ & $9^{\circ} 45^{\prime} 10,5^{\prime \prime}$ & $9^{\circ} 45^{\prime} 08,2^{\prime \prime}$ & $9^{\circ} 45^{\prime} 09,8^{\prime \prime}$ & $9^{\circ} 45^{\prime} 10,2^{\prime \prime}$ \\
\hline \multirow[t]{3}{*}{ Bonamoussadi } & & $\mathrm{C} 1$ & $\mathrm{C} 2$ & $\mathrm{C} 3$ & $\mathrm{C} 4$ & $\mathrm{C} 5$ & C6 & C7 \\
\hline & $\mathrm{X}$ & $4^{\circ} 05^{\prime} 03,2^{\prime \prime}$ & $4^{\circ} 05^{\prime} 5,1^{\prime \prime}$ & $4^{\circ} 05^{\prime} 11,7^{\prime \prime}$ & $4^{\circ} 05^{\prime} 10,3^{\prime \prime}$ & $4^{\circ} 05^{\prime} 16,7^{\prime \prime}$ & $4^{\circ} 05^{\prime} 17,6^{\prime \prime}$ & $4^{\circ} 05^{\prime} 19,3^{\prime \prime}$ \\
\hline & $\mathrm{Y}$ & $9^{\circ} 44^{\prime} 12,4^{\prime \prime}$ & $9^{\circ} 44^{\prime} 30,8^{\prime \prime}$ & $9^{\circ} 44^{\prime} 36,8^{\prime \prime}$ & $9^{\circ} 44^{\prime} 23,1^{\prime \prime}$ & $9^{\circ} 44^{\prime} 38,2^{\prime \prime}$ & $9^{\circ} 44^{\prime} 40,3^{\prime \prime}$ & $9^{\circ} 44^{\prime} 39,5^{\prime \prime}$ \\
\hline \multirow[t]{3}{*}{ Bonapriso } & & E1 & E2 & E3 & $\mathrm{E} 4$ & E5 & E6 & E7 \\
\hline & $\mathrm{X}$ & $4^{\circ} 01^{\prime} 36,5^{\prime \prime}$ & $4^{\circ} 01^{\prime} 37,8^{\prime \prime}$ & $4^{\circ} 01^{\prime} 29,3^{\prime \prime}$ & $4^{\circ} 01^{\prime} 31,4^{\prime \prime}$ & $4^{\circ} 01^{\prime} 22,6^{\prime \prime}$ & $4^{\circ} 01^{\prime} 26,9^{\prime \prime}$ & $4^{\circ} 01^{\prime} 33,1^{\prime \prime}$ \\
\hline & $\mathrm{Y}$ & $9^{\circ} 44^{\prime} 19,7^{\prime \prime}$ & $9^{\circ} 44^{\prime} 21,5^{\prime \prime}$ & $9^{\circ} 44^{\prime} 22,3^{\prime \prime}$ & $9^{\circ} 44^{\prime} 24,5^{\prime \prime}$ & $9^{\circ} 44^{\prime} 21,3^{\prime \prime}$ & $9^{\circ} 44^{\prime} 25,8^{\prime \prime}$ & $9^{\circ} 44^{\prime} 28,2^{\prime \prime}$ \\
\hline
\end{tabular}


Tableau 3: Description de l'environnement physique des différents points d'eau prélevés et les usages de l'eau.

\begin{tabular}{|c|c|c|c|}
\hline Groupe & Puits & Présentation de l'environnement physique & Usages \\
\hline A & $\begin{array}{l}\mathrm{B}_{3}, \mathrm{~B}_{5}, \mathrm{M}_{2}, \\
\mathrm{~N}_{5}, \mathrm{~N}_{7}, \mathrm{P}_{5}\end{array}$ & $\begin{array}{l}\text { situé entre } 5 \text { et } 10 \mathrm{~m} \text { de la fosse d'aisances et autres } \\
\text { sources de pollution, d'une profondeur de } 0,5 \text { à } 2 \mathrm{~m} \text {, } \\
\text { sans traitement, aucun entretien dans les alentours }\end{array}$ & $\begin{array}{l}\text { Baignade, lessive, } \\
\text { vaisselle et } \\
\text { cuisson des repas }\end{array}$ \\
\hline \multirow[t]{2}{*}{ B } & $\begin{array}{l}\mathrm{D}_{7}, \mathrm{~N}_{1}, \mathrm{P}_{1} \\
\mathrm{D}_{6}, \mathrm{M}_{1}, \mathrm{P}_{4} \\
\mathrm{P}_{7}, \mathrm{~B}_{4}, \mathrm{M}_{7} \\
\mathrm{C}_{6}, \mathrm{~B}_{6}\end{array}$ & $\begin{array}{l}\text { situé entre } 5 \text { et } 10 \mathrm{~m} \text { de la fosse d'aisances et autres } \\
\text { sources de pollution, d'une profondeur de } 2 \text { à } 4 \mathrm{~m} \text {, } \\
\text { pas de traitement à l'eau de javel }\end{array}$ & \multirow{2}{*}{$\begin{array}{l}\text { Cuisson des repas, } \\
\text { baignade, } \\
\text { vaisselle et lessive }\end{array}$} \\
\hline & $\begin{array}{l}\mathrm{M}_{3}, \mathrm{M}_{4}, \mathrm{~N}_{6} \\
\mathrm{P}_{3}\end{array}$ & $\begin{array}{l}\text { situé entre } 10 \text { et } 15 \mathrm{~m} \text { de la fosse d'aisances et autres } \\
\text { sources de pollution, d'une profondeur de } 0,5 \text { à } 2 \mathrm{~m} \text {, } \\
\text { bénéficie d'un traitement à l'eau de javel }\end{array}$ & \\
\hline \multirow{3}{*}{$\mathrm{C}$} & $\begin{array}{l}\mathrm{B}_{1}, \mathrm{~B}_{7}, \mathrm{C}_{2}, \mathrm{D}_{2} \\
\mathrm{M}_{5}, \mathrm{M}_{6}, \mathrm{~N}_{3} \\
\mathrm{P}_{2}, \mathrm{P}_{6}\end{array}$ & $\begin{array}{l}\text { situé à plus de } 15 \mathrm{~m} \text { de la fosse d'aisances et autres } \\
\text { sources de pollution, d'une profondeur de } 2 \text { à } 5 \mathrm{~m} \text {, } \\
\text { traitement mensuel à l'eau de javel, alentours bien } \\
\text { entretenus }\end{array}$ & \multirow{3}{*}{$\begin{array}{l}\text { Baignade, } \\
\text { vaisselle, cuisson } \\
\text { des repas, lessive }\end{array}$} \\
\hline & $\mathrm{D}_{5}, \mathrm{E}_{3}, \mathrm{E}_{4}, \mathrm{~N}_{6}$ & $\begin{array}{l}\text { Situé à plus de } 15 \mathrm{~m} \text { des sources de pollution, d'une } \\
\text { profondeur de moins de } 2 \mathrm{~m} \text {, bénéficie d'un } \\
\text { traitement à l'eau de javel }\end{array}$ & \\
\hline & $\begin{array}{l}D_{1}, D_{3}, D_{4} \\
C_{3}\end{array}$ & $\begin{array}{l}\text { situé à plus de } 15 \mathrm{~m} \text { des sources de pollution, d'une } \\
\text { profondeur de } 8 \text { à } 15 \mathrm{~m} \text {, bénéficie d'un traitement à } \\
\text { l'eau de javel }\end{array}$ & \\
\hline \multirow{5}{*}{$\mathrm{D}$} & $\mathrm{E}_{2}, \mathrm{E}_{5}, \mathrm{E}_{7}$ & $\begin{array}{l}\text { Situé à plus de } 20 \mathrm{~m} \text { des sources de pollution, d'une } \\
\text { profondeur de } 2 \text { à } 4 \mathrm{~m} \text {, bénéficie d'un traitement à } \\
\text { l'eau de javel }\end{array}$ & \multirow{5}{*}{$\begin{array}{l}\text { Baignade, } \\
\text { vaisselle, cuisson } \\
\text { des repas, lessive }\end{array}$} \\
\hline & $\mathrm{N}_{2}$ et $\mathrm{N}_{4}$ & $\begin{array}{l}\text { Situé à plus de } 20 \mathrm{~m} \text { des sources de pollution, d'une } \\
\text { profondeur de } 4 \text { à } 6 \mathrm{~m} \text {, ne bénéficie pas de traitement } \\
\text { à l'eau de javel }\end{array}$ & \\
\hline & $\begin{array}{l}\mathrm{E}_{1}, \mathrm{E}_{6}, \mathrm{C}_{4} \text { et } \\
\mathrm{C}_{5}\end{array}$ & $\begin{array}{l}\text { Situé à plus de } 20 \mathrm{~m} \text { des sources de pollution, d'une } \\
\text { profondeur de } 4 \text { à } 6 \mathrm{~m} \text {, bénéficie d'un traitement à } \\
\text { l'eau de javel }\end{array}$ & \\
\hline & $\mathrm{C}_{1}, \mathrm{C}_{7}$ & $\begin{array}{l}\text { Situé à plus de } 20 \mathrm{~m} \text { des sources de pollution, d'une } \\
\text { profondeur de } 6 \text { à } 10 \mathrm{~m} \text {, bénéficie d'un traitement à } \\
\text { l'eau de javel }\end{array}$ & \\
\hline & $\mathrm{B}_{2}$ & $\begin{array}{l}\text { Situé en altitude par rapport à une fosse d'aisance ; } \\
\text { surmonté d'une margelle en béton, sa profondeur est } \\
\text { de } 27 \mathrm{~m} \text {, alentours bien entretenus, bénéficie d'un } \\
\text { traitement à l'eau de javel trimestriellement. }\end{array}$ & \\
\hline
\end{tabular}




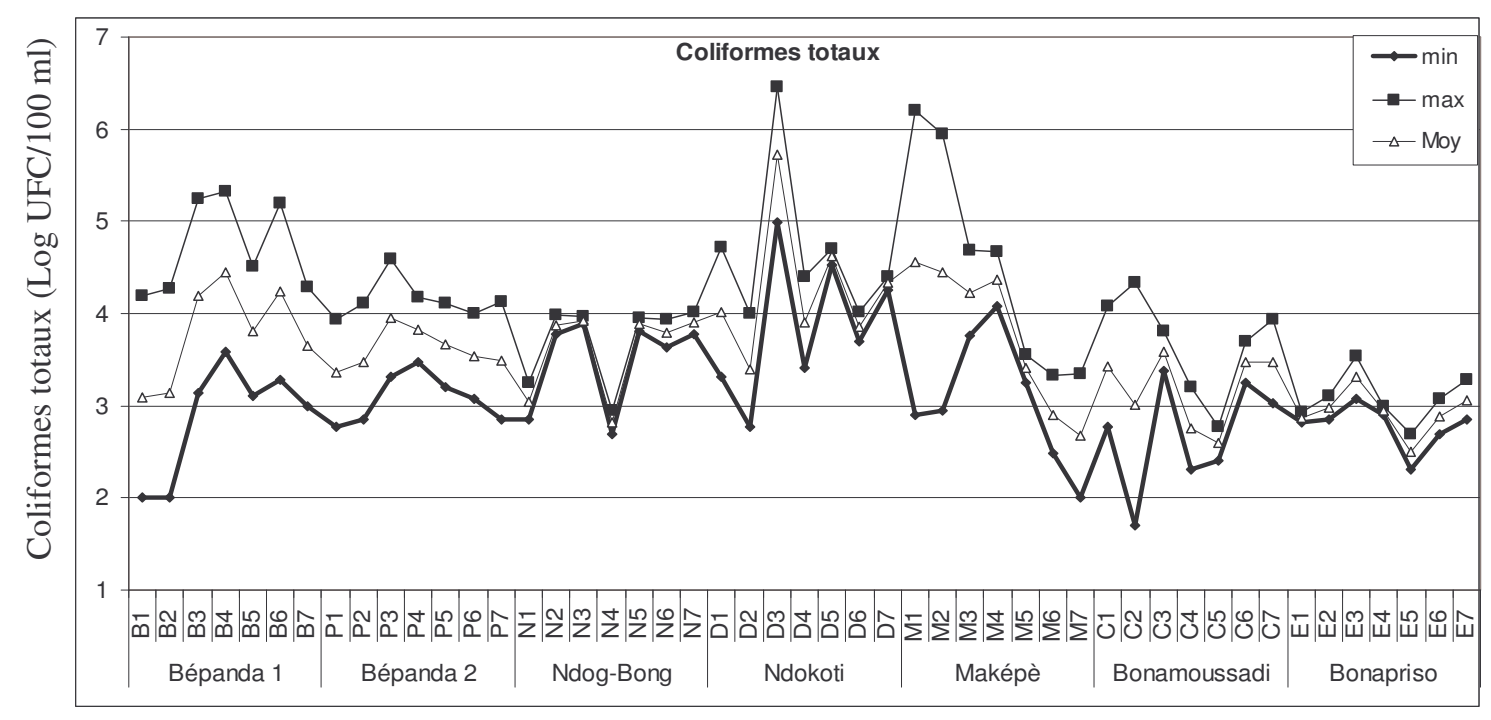

Figure 2 : Valeurs logarithmiques moyennes, maximales et minimales des coliformes totaux dans les eaux des puits analysées à Douala (moyenne sur 12 analyses réalisées en 12 semaines).

Tableau 4: Teneurs en coliformes totaux et fécaux (moyenne sur 84 échantillons: 7 puits échantillonnés 12 fois en 12 semaines).

\begin{tabular}{lcccc}
\hline & \multicolumn{2}{c}{ Coliformes totaux } & \multicolumn{2}{c}{ Coliformes fécaux } \\
\hline & Moyenne en UFC & Ecart Type & Moyenne en UFC & Ecart Type \\
\hline Bépanda 1 (B) & $4,58 \mathrm{E}+04$ & $4,40 \mathrm{E}+04$ & $9,06 \mathrm{E}+03$ & $9,22 \mathrm{E}+03$ \\
\hline Bépanda 2 (P) & $8,71 \mathrm{E}+03$ & $5,39 \mathrm{E}+03$ & $1,71 \mathrm{E}+02$ & $9,09 \mathrm{E}+01$ \\
\hline Ndog-Bong (N) & $5,82 \mathrm{E}+03$ & $3,38 \mathrm{E}+03$ & $2,14 \mathrm{E}+02$ & $1,50 \mathrm{E}+02$ \\
\hline Ndokoti (D) & $2,31 \mathrm{E}+05$ & $5,60 \mathrm{E}+05$ & $3,34 \mathrm{E}+02$ & $3,44 \mathrm{E}+02$ \\
\hline Maképè Missoquè (M) & $1,85 \mathrm{E}+05$ & $3,12 \mathrm{E}+05$ & $7,42 \mathrm{E}+02$ & $1,28 \mathrm{E}+03$ \\
\hline Bonamoussadi (C) & $4,44 \mathrm{E}+03$ & $3,52 \mathrm{E}+03$ & $3,42 \mathrm{E}+02$ & $2,66 \mathrm{E}+02$ \\
\hline Bonapriso (E) & $1,07 \mathrm{E}+03$ & $6,32 \mathrm{E}+02$ & $4,21 \mathrm{E}+01$ & $1,22 \mathrm{E}+01$ \\
\hline
\end{tabular}

concentrations des bactéries isolées, que tous les points d'eau des zones insalubres d'une part et des zones «haut standing» d'autre part, diffèrent entre eux. Aussi, la différence des moyennes de densités bactériennes entre les zones insalubres et les zones mieux loties est significative $(p<0,05)$. Selon le test de Fischer, on peut grouper les puits en 3 catégories: la première catégorie constitue les puits $\mathrm{B}_{3}, \mathrm{~B}_{4}$, $\mathrm{B}_{6}, \mathrm{~B}_{7}, \mathrm{M}_{2}, \mathrm{M}_{3}$ où la contamination fécale est très forte, la deuxième catégorie forme les puits du groupe $\mathrm{C}$ (Tableau 3 ) où les densités de coliformes fécaux sont faibles, les maxima étant moins de $500 \mathrm{UFC} / 100 \mathrm{ml}$, et enfin la troisième catégorie concerne les puits $\mathrm{N}_{4}, \mathrm{C}_{5}$, $\mathrm{E}_{1}$ à $\mathrm{E}_{7}$ dont les densités maximales de coliformes fécaux sont en majorité autour de 30 UFC / 100ml. Ainsi la première catégorie de puits diffère très significativement de la troisième catégorie $(\mathrm{p}<0,01)$, et de la deuxième catégorie $(\mathrm{p}<0,05)$; les eaux de puits de la deuxième catégorie diffèrent aussi de celles de la troisième catégorie $(\mathrm{p}<0,05)$.

Les streptocoques fécaux ont été évalués par leur présence ou absence. La nécessité de détecter leur présence ou absence est due au 


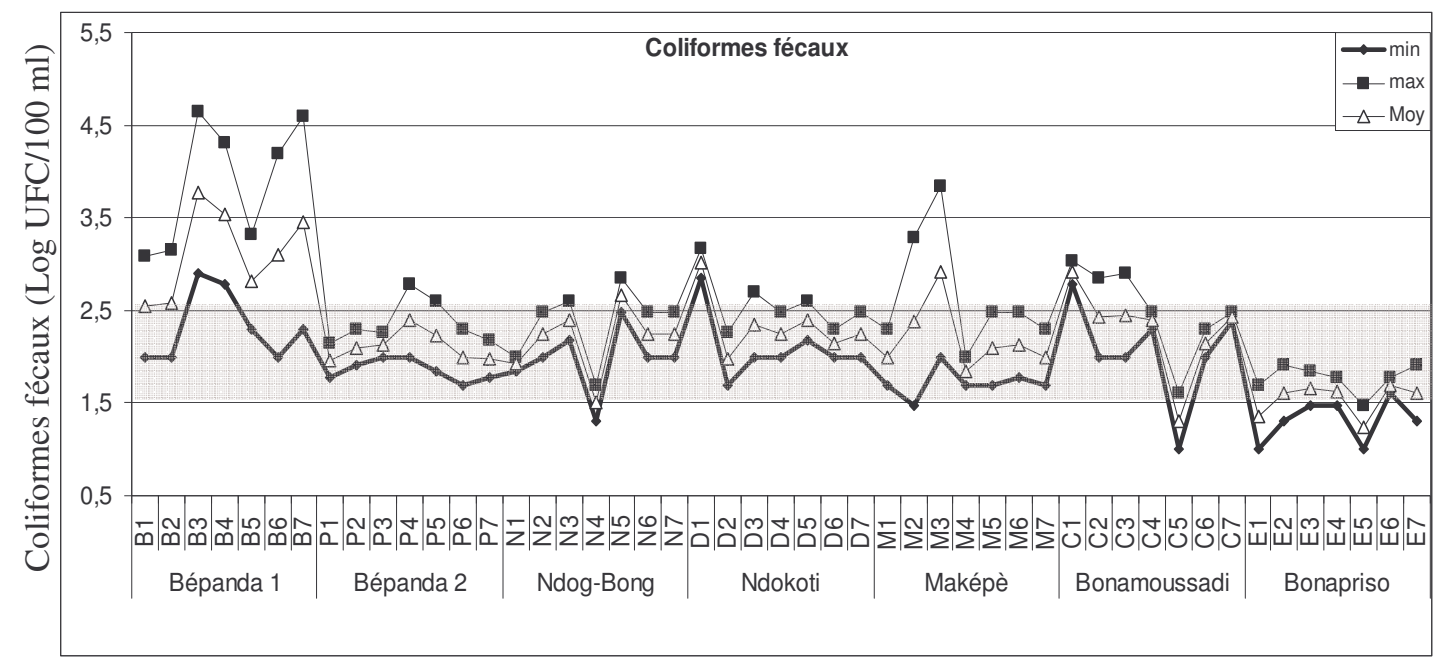

Figure 3: Valeurs logarithmiques moyennes, maximales et minimales des coliformes fécaux dans les eaux des puits analysées à Douala (moyenne sur 12 analyses réalisées en 12 semaines). La zone colorée regroupe le maximum de puits du groupe C (Tableau 2). Les eaux des groupes A et B sont au dessus de cette zone; celles du groupe $\mathrm{D}$ en dessous.

fait que les coliformes fécaux témoignent d'une contamination fécale récente, et les streptocoques fécaux témoignent d'une contamination fécale ancienne (Trevisan et al., 2002). Les streptocoques fécaux sont partout présents sauf dans un puits à Ndog-Bong $\left(\mathrm{D}_{4}\right)$, un à Bonamoussadi $\left(\mathrm{C}_{5}\right)$ et un autre à Bonapriso $\left(\mathrm{E}_{5}\right)$. La caractéristique de ces puits exemptés de streptoccoques est qu'ils sont dans des quartiers assez propres (haut-standing), moins peuplés, organisés suivant un schéma directeur adéquat. Aussi, ces puits sont situés à plus de $15 \mathrm{~m}$ de la source de pollution, sont javellisés et le niveau de la nappe est à plus de $4 \mathrm{~m}$ de profondeur.

\section{Variation de la teneur en bactéries des puits de Douala}

Il ressort des figures 2 et 3 que les puits les moins pollués ont une faible amplitude de fluctuation des germes. Dans l'ensemble, ces puits doivent être sujets à une contamination permanente provenant des sources de pollution non éloignées et à leur faible profondeur (Tableau 3). Les puits à fort taux de pollution par les bactéries pathogènes présentent aussi les fortes amplitudes de variations de densités de ces germes.

\section{DISCUSSION}

L'abondance des coliformes fécaux et la présence des streptocoques fécaux dans les eaux des puits de Douala y est influencée par la profondeur totale du puits, l'épaisseur de la colonne d'eau, de la qualité initiale de l'eau et $\mathrm{du}$ traitement qui $\mathrm{y}$ est fait, et des aménagements faits autour du puits. On peut aussi constaté que tous les puits d'un même groupe (Tableau 3) ne se retrouvent pas forcément dans la même catégorie de potabilité.

Les puits qui présentent les plus fortes densités de germes sont ceux des groupes A et B (Tableau 3), puits des quartiers Ndokoti, Maképè Missoquè et Bépanda 1 principalement. Ils sont caractérisés par le niveau de l'eau proche de la surface $(0,5$ à 4 $\mathrm{m})$, la proximité des latrines (moins de $10 \mathrm{~m}$ ), l'absence d'entretien des alentours et du traitement. Selon Boutin (1987), Nola et al. (2000a), l'eau d'une nappe phréatique apparaît généralement d'autant plus vulnérable que le sommet de la nappe phréatique est proche de la surface du sol, que les terrains qui surmontent l'aquifère sont perméables et que les sources superficielles de pollution sont importantes, nombreuses et proches du site d'étude. Les résultats obtenus dans cette étude confirment le 
mauvais environnement de ces puits. Le mauvais entretien serait à l'origine des pollutions de certains puits. Ces résultats corroborent ceux trouvés dans d'autres quartiers populaires. Bemmo et al. (1998) dans la ville de Yaoundé notent des teneurs pouvant atteindre $37 * 10^{4}$ de coliformes totaux et $35^{*} 10^{2}$ UFC/100 ml en coliformes fécaux, avec les teneurs les plus élevées obtenues dans les puits dépourvus de couvercle. Les teneurs, encore plus élevées, de 3 à $4 * 10^{6} \mathrm{UFC} / 100 \mathrm{ml}$ de coliformes totaux et $1,15^{*} 10^{4}$ de coliformes fécaux, ont aussi été obtenues dans les eaux des puits non aménagés dans la ville de Dschang (Temgoua et Kuetche, 2009). Ndjama et al. (2008) dans certains de ces quartiers à Douala ont montré que l'eau est, pour $50 \%$ de ménages, sollicitée dans les puits.

Malgré que les puits $\mathrm{B}_{7}, \mathrm{C}_{4}, \mathrm{D}_{1}$ et $\mathrm{M}_{3}$ subissent de temps à autre un traitement à l'eau de javel, leurs eaux restent de mauvaise qualité. Alors, soit la quantité d'eau de javel utilisée pour la désinfection n'est pas proportionnelle au volume d'eau dans le puits, soit la fréquence de traitement n'est pas appropriée. En outre, il peut aussi avoir dans ces eaux des substances azotées $\left(\mathrm{NH}_{4}{ }^{+}\right)$qui se combinent avec le chlore de l'eau de javel pour former le chloramine et le rendent beaucoup moins efficace (Montiel, 1982). La survie des microorganismes peut être liée au développement par ces germes d'une gamme de stratégies qui, selon le cas, peuvent aller de l'acquisition de plasmides aux changements de voies métaboliques en passant par les mutations génétiques (Chao et Feng, 1990). Déjà, Niquette et al. (2001) recommandent qu'en cas de contamination microbiologique d'une eau souterraine, la désinfection soit faite après avoir détecté et éliminé la source de pollution. Selon Beauchamp (2003), la quantité d'eau de javel requise pour la désinfection varie en fonction de la concentration bactérienne décelée après étude, du volume d'eau dans le puits et selon que celui-ci soit régulièrement traité.

Les puits qui présentent le faible taux de pollution par les bactéries (troisième catégorie) se retrouvent dans les groupes $\mathrm{C}$ et D (Tableau 3) des quartiers Ndog-Bong, Bepanda 2, Bonamoussadi et Bonapriso. Ces puits sont aménagés, munis d'un couvercle bien utilisé et d'une margelle en béton, avec une profondeur d'au moins $4 \mathrm{~m}$, situés au moins à $15 \mathrm{~m}$ en amont d'une quelconque source de pollution et dont le traitement est régulier. Les contaminations fécales y sont inférieures à celles des autres puits d'au moins 1000 unités. Les normes OMS (1996) ne tolèrent aucune UFC/100 ml d'eau; cependant, tenant compte des contraintes locales, Duchemin (1998) propose des valeurs de $20 \mathrm{UFC} / 100 \mathrm{ml}$ d'eau comme limites acceptables en Afrique subsaharienne. Les puits des groupes $\mathrm{C}$ et $\mathrm{D}$ présentent donc une qualité bactériologique acceptable pour une eau à usage humaine.

La variation des bactéries fécales dans l'eau des puits étudiés serait aussi dues aux facteurs pédologiques et aux caractéristiques de la nappe phréatique comprenant entre autres, le caractère saturé ou insaturé du sol, sa porosité et la nature des substances chimiques présentes dans ces eaux (Bemmo et al., 1998 ; Nola et al., 2000b). L'assainissement des eaux pluviales et usées de ces quartiers reste une des préoccupations majeures (Djuikom et al., 2006). Si la topographie de Douala en est un facteur aggravant, le mauvais écoulement des eaux résulte également du mauvais comportement des ménages face à l'évacuation des déchets solides (rejets sauvages conduisant à l'obstruction des drains) et de l'urbanisation désordonnée (Urbaplan et Sogreah, 2006). Il existe tout de même un rapport étroit entre l'abondance de coliformes fécaux et la présence de streptocoques fécaux (Djuikom et al., 2006). En effet, les deux groupes de germes sont des hôtes du tube digestif, à la différence que les coliformes fécaux témoignent d'une contamination fécale récente alors que les streptocoques fécaux, plus résistants dans l'environnement, témoignent d'une contamination fécale ancienne (Trevisan et al., 2002). Au sujet de la variation de la densité des germes, on peut penser pour les fortes variations de germes à un changement des habitudes lié à une hygiène appropriée, ou un traitement récent qui aurait précédé certains prélèvements. Par ailleurs, il doit y avoir de manière temporelle des apports exogènes ou une quelconque infiltration ponctuelle de polluants jusqu'à la nappe phréatique dans les puits des zones insalubres. 


\section{Conclusion}

Les résultats de cette étude montrent que les eaux de puits à Douala hébergent de nombreuses communautés de germes indicateurs de contamination fécale, parmi lesquelles, les coliformes fécaux et les streptocoques fécaux. Leurs densités subissent d'importantes fluctuations spatiales et temporelles, et varient entre 100 et $29 * 10^{5}$ $\mathrm{UFC} / 100 \mathrm{ml}$ pour les coliformes totaux et de 30 à $44,8 * 10^{3} \mathrm{UFC} / 100 \mathrm{ml}$ pour les coliformes fécaux; les streptocoques fécaux sont très souvent présents. Les facteurs de variations sont l'environnement du puits, l'entretien, le niveau de la nappe dans le puits, la situation géographique. Les puits les plus contaminés (première catégorie) sont ceux qui sont situés à moins de $5 \mathrm{~m}$ des sources de pollution telles que les latrines à fond perdu, les dépôts sauvages des ordures ménagères, l'absence ou le traitement irrégulier de l'eau, le niveau superficiel de l'eau dans le puits. Les puits aménagés (des groupes $\mathrm{C}$ et $\mathrm{D}$ ), c'est-à-dire munis d'un couvercle bien utilisé et d'une margelle en béton, avec une profondeur d'au moins $4 \mathrm{~m}$, situés au moins à $15 \mathrm{~m}$ en amont d'une quelconque source de pollution et dont le traitement est régulier, présentent une eau de qualité beaucoup améliorée; les contaminations fécales y baissent d'au moins 1000 unités. Les points d'eau non aménagés sont très nombreux et les fortes densités de germes dans ces eaux montrent de grands risques sanitaires à court terme pour les consommateurs. Les autorités municipales et les ONG s'activent sur le terrain mais aucune action n'a encore été entreprise pour améliorer la qualité de ces eaux pourtant consommées par les populations.

Dans l'incertitude de pouvoir assainir parfaitement les quartiers où les puits sont pollués, l'accès à l'eau potable apparaît clairement comme une priorité pour endiguer les maladies d'origine hydrique, notamment le choléra. Ces résultats permettent de proposer aux populations, en l'absence totale du réseau conventionnel, de ne pas creuser les puits proches des sources de contamination (latrines à fond perdu, dépôts d'ordures, milieu marécageux), mais plutôt de désinfecter l'eau des puits au moins une fois tous les mois à l'eau de javel et de demander les conseils aux ingénieurs sanitaires pour la quantité d'eau de javel à utiliser, car celle-ci doit être proportionnelle au volume d'eau dans le puits et de la fréquence de traitement.

\section{REMERCIEMENTS}

Les auteurs remercient la Fondation Internationale pour la Science (IFS), bourse W/4201-1, pour le soutien à la recherche qui a permis la réalisation de ce travail.

\section{REFERENCES BIBLIOGRAPHIQUES}

Beauchamp J. 2003. Qualité et pollution des eaux souterraines. Rapport interne, Université de Picardie Jules Verne, 10 p.

Bemmo N, Njine T, Nola M, Ngamga D. 1998. Techniques utilisées au niveau des quartiers périurbains de Yaoundé (Cameroun) pour l'évacuation des eaux usées et excréta humains; propositions de systèmes appropriés. pS-Eau-Coop Française. Rapport final Action de recherche $\mathrm{n}^{\circ}$ 4, ENSP de Yaoundé, $126 \mathrm{p}$.

Boutin C. 1987. L'eau des nappes phréatiques superficielles, une richesse naturelle vitale mais vulnérable. L'exemple des zones rurales du Maroc. Sciences de l'Eau, 6: $357-365$.

Chao WL, Feng RL. 1990. Survival of genetically engineered Escherichia coli in natural soil and river water. J. Appl. Bacteriol., 68: 319-325.

Délégation de la Santé du Littoral, 2004. Epidémie de choléra à Douala, Cameroun. Direction des Collectivités Publiques.

Djeuda Tchapnga HB, Tanawa E, Ngnikam E. 2001. L'eau au Cameroun. Tome 1: approvisionnement en eau potable. Presses Universitaires de Yaoundé; 359 p.

Djuikom E, Njiné T, Nola M, Sikati V, Jugnia LB. 2006. Microbiological Water quality of the Mfoundi at Yaounde, Cameroon, as inferred from indicator bacteria of fecal contamination. Envir. Monitoring Assessment, 122: 171-183.

Duchemin JP. 1998. Impact des conditions d'alimentation en eau potable et d'assainissement sur la santé publique. Document de synthèse pS-Eau, Ouagadougou, avril 1998, 16 p. 
INS. 2005. Annuaire Statistique du Cameroun 2004. Institut National de Statistique.

Jain P, Sharma JD, Sohu D, Sharma P. 2005. Chemical analysis of drinking water of villages of Sanganer Tehsil, Jaipur District. Int. J. Environ. Sci. Tech., 2/4: 373-379.

Kreisel W. 1991. Water quality and health. Wat. Sci. Tech., 23: 201-209.

Leclerc H. 1986. Pathologie liée à l'usage de l'eau. Aqua, 6: 329-333.

Marchal N, Bourdon JL, Richard CL. 1991. Les milieux de culture pour l'isolement et l'identification biochimique des bactéries. Paris : Doin, 509 p.

Montiel AJ. 1982. Les principes à observer en matière de contrôle des eaux d'alimentation. Service du Contrôle des Eaux de la Ville de Paris ; 34-40.

Moshtaghi H, Boniadian M. 2007. Microbial quality of drinking water in Sharekord (Iran). Research Journal of Microbiology, 2/3: 299-302.

Ndjama J, Kamgang Kabeyene BV, Sigha Nkamdjou L, Ekodeck G, Tina M Awah 2008. Water supply, sanitation and health risks in Douala, Cameroon. African Journal of Environmental Science and Technology, 2(12): 422-429.

Niquette P, Servais P, Savoir R. 2001. Bacterial dynamics in the drinking water distribution system of Brussels. Water Resources, 35(3): 675-682.

Nola M, Njine T, Boutin C. 1998. Variabilité de la qualité des eaux souterraines dans quelques stations de Yaoundé (Cameroun). Mémoire Biospéol., 25: 183191.

Nola M., Njine T., Djuikom E. et Sikati Foko V. 2000a. Bacteria indicators dynamics in wells as influenced by well depth and well water column thickness, in Yaounde (Cameroon). African Journal of Environmental Science and Technology, 1: 82-91.

Nola M, Njine T, Djuikom E, Sikati Foko V. 2000b. Faecal coliforms and faecal streptococci community in the underground water in an equatorial area in Cameroon (Central Africa): the importance of some environmental chemical factors. Water Research, 36: 3289-3297.

OMS. 1996. Directives de Qualité pour l'Eau de Boisson (Vol. 2, 2e édn). OMS : Genève.

Ousmane B, Daddy Gao A, Soumaila A, Margueron T, Boubacar A, Garba Z, Ousmane S. 2007. Origine de la pollution des nappes superficielles et profondes au niveau de la communauté urbaine de Niamey (Niger). Africa Geoscience Review, 14(3): 375-398.

Palmateer GA, Dutka BJ, Janzen EM, Meissner SM, Sakellaris MG. 1991. Coliphage and bacteriophage as indicators of recreational water quality. Water Resources, 25: 355357.

Schwartz D. 1984. Méthodes Statistiques à l'Usage des Médecins et des Biologistes. Flammarion : Paris ; 318p.

Temgoua E, Ngnikam E, Ndongson B 2009. Drinking water quality: stakes of control and sanitation in the town of Dschang Cameroon. International Journal of Biological and Chemical Sciences, 3(3): 441-447.

Temgoua E, Kuetche S. 2009. Analyse de la qualité des eaux des points aménagés en 2008 par l'AIMF dans la ville de Dschang. Rapport GIE-UDs, 26 pages.

Trevisan D, Vansteelant JY, Dorioz JM. 2002. Survival and leaching of faecal bacteria after slurry spreading on mountain hay meadows: consequences for the management of water contamination risk. Water Research, 36: 275-283.

URBAPlAn, SOGREAH. 2006. Etude du schéma directeur d'assainissement de la ville de Douala et maîtrise d'œuvre d'une tranche prioritaire: étude d'un programme d'aménagement et d'assainissement des quartiers précaires de la ville de Douala. Phase 2 : Diagnostic et stratégie. Rapport Urbaplan, $56 \mathrm{p}$. 